\title{
RELAÇÕES ALOMÉTRICAS EM ÁRVORES DE Pterodon emarginatus VOGEL EM UM SISTEMA SILVIPASTORIL
}

\author{
Omar Daniel ${ }^{1}$, Thobias Pezzoni ${ }^{2}$, Igor Murilo Bumbieris Nogueira ${ }^{3}$, Thais Cremon ${ }^{4}$, Antonio Carlos Tadeu Vitorino ${ }^{5}$
}

(recebido: 13 de junho de 2010; aceito: 28 de outubro de 2012)

RESUMO: Relações alométricas podem ser aplicadas na previsão da densidade ideal de árvores, nas avaliações de competição, nas inferências sobre a estabilidade, vitalidade e produtividade e na elaboração de modelos de crescimento florestal. Árvores em consórcio com forrageiras, os sistemas silvipastoris (SSP), são cada vez mais frequentes, considerando as vantagens do componente arbóreo para a pastagem e animais. Neste estudo, objetivou-se avaliar por meio de regressão, as relações alométricas possíveis entre diâmetro a 1,3 m de altura (DAP), altura de copa (Ac), altura de fuste (Af), altura total (At), diâmetro (Dc) e volume cilíndrico de copa (Vc) em um SSP com Pterodon emarginatus, como subsidio ao seu manejo. Os dados foram coletados na região de Nioaque - MS, em área de consórcio P. emarginatus x B. decumbens x bovinos de corte, no qual as árvores foram mantidas intactas durante as operações de desmatamento. Para a regressão, foram coletados aleatoriamente dados de 91 indivíduos adultos de variadas dimensões. Os modelos foram avaliados por análise de resíduos, significância dos coeficientes de regressão, coeficiente de determinação e erro padrão residual. $\mathrm{O}$ modelo potência de Stoffels foi o mais adequado para estimar a At $f(\mathrm{DAP})$; a reta resultou nos melhores níveis de precisão para as estimativas de Ac $f(\mathrm{At}), \mathrm{Vc} f$ (DAP) e Dc $f$ (DAP); foram obtidos bons níveis de precisão para estimar Vc $f$ (DAP; At) por meio de um dos modelos de Spurr $\left[\mathrm{y}=\mathrm{a}+\mathrm{b}\right.$.(DAP ${ }^{2}$. At $\left.)\right]$.

Palavras-chave: Sistemas agroflorestais, relações hipsométricas, árvores em pastagens.

\section{ALLOMETRIC RELATIONS ON Pterodon emarginatus VOGEL TREES IN A SILVOPASTORAL SYSTEM}

ABSTRACT: Allometric relations can be applied in the forecast of the ideal density of trees, competition evaluations, inferences about the stability, vitality and productivity and in the elaboration of forest growth models. Trees in consortium with forages, the silvopastoral systems (SSP), are very frequent nowadays, considering the advantages of the arboreal component for the pasture and animals. It support the present study with objective to evaluate, under the regression form, the possible relationships among diameter at breast height (DAP), crown height (Ac), stem height $(A f)$, total height $(A t)$, diameter $(D c)$ and cylindrical volume of crown $(V c)$ in a SSP with Pterodon emarginatus, subsidizing their management. The data were collected in Nioaque - MS county, in a consortium area of $P$. emarginatus $x$ B. decumbens $x$ beef cattle, in which the trees were maintained intact during deforestation. For the regression, data of 91 adult trees of varied dimensions were randomly collected. The models were evaluate for graphical analysis of residues, significance of the regression coefficients, coefficient of determination and residual standard error. The Stoffels potential model was more appropriate for estimate At $f(D A P)$; the straight line resulted on the betters levels to estimate Ac $f(A t), V c f(D A P)$ e Dc $f(D A P)$; good fitness to estimate Vcf $(D A P ; A t)$ were obtained from one of Spurr model $\left[y=a+b .\left(D A P^{2} . A t\right)\right]$.

Key words: Agroforestry systems, hypsometric relations, tree on pastures.

\section{INTRODUÇÃo}

No Mato Grosso do Sul (MS), encontram-se dois dos seis biomas brasileiros, o Cerrado e a Mata Atlântica, ambos incluídos na lista mundial de hotspots (MITTERMEIER et al., 2005), em função da sua rica e endêmica biodiversidade, contrastada com a perda de mais de $75 \%$ de suas superfícies. No MS, assenta-se também um grande número de propriedades rurais cujas atividades econômicas estão voltadas à agropecuária, demonstrando que a descaracterização desses biomas na região ocorreu, predominantemente, pela expansão da fronteira agrícola, gerando, além de outros problemas ambientais, a fragmentação da vegetação.

\footnotetext{
${ }^{1}$ Engenheiro Florestal, Professor Doutor em Ciência Florestal - Universidade Federal da Grande Dourados - Faculdade de Ciências Agrárias - Rua João Rosa Góes, 1761, Vila Progresso - Cx. P. 322 - 79.825-070 - Dourados, MS, Brasil - omar.daniel@pq.cnpq.br ${ }^{2}$ Engenheiro Agrônomo, Mestre em Agronomia - Universidade Federal da Grande Dourados - Faculdade de Ciências Agrárias - Rua João Rosa Góes, 1761, Vila Progresso - Cx. P. 322 - 79.825-070 - Dourados, MS, Brasil - thobiasburi@bol.com.br ${ }^{3}$ Engenheiro Agrônomo, Mestrando em Agronomia - Universidade Federal da Grande Dourados - Faculdade de Ciências Agrárias - Rua João Rosa Góes, 1761, Vila Progresso - Cx. P. 322 - 79.825-070 - Dourados, MS, Brasil - igorbumbieris@hotmail.com ${ }^{4}$ Engenheira Agrônoma, Mestranda em Agronomia - Universidade Federal da Grande Dourados - Faculdade de Ciências Agrárias - Rua João Rosa Góes, 1761, Vila Progresso - Cx. P. 322 - 79.825-070 - Dourados, MS, Brasil - thaiscremon@ hotmail.com ${ }^{5}$ Engenheiro Agrônomo, Professor Doutor em Ciência do Solo - Universidade Federal da Grande Dourados - Faculdade de Ciências Agrárias - Rua João Rosa Góes, 1761, Vila Progresso - Cx. P. 322 - 79.825-070 - Dourados, MS, Brasil - antoniovitorino@ufgd.edu.br
} 
Na expansão da pecuária sobre o Centro-Oeste, a forma mais utilizada para a formação das pastagens consistiu na erradicação total da vegetação nativa, substituindo-a por gramíneas de origem africana, em especial do gênero Brachiaria. No entanto, em muitas regiões onde a leguminosa arbórea Pterodon spp. se distribui, como é o caso de grandes extensões de terra no MS (Nioaque, Guia Lopes da Laguna, Bonito e Anastácio), tornou-se hábito dos pecuaristas manter indivíduos dessa espécie formando um sistema silvipastoril (SSP) intuitivo.

A tradição de se manter árvores do gênero Pterodon no sistema de produção pecuária está associada aos benefícios ambientais gerados, como a sombra para os animais e a proteção ambiental (DANIEL et al., 1999). Uma das espécies desse gênero, a sucupirabranca, também conhecida por faveira ( $P$. emarginatus Vogel), é típica de regiões de transição entre o Cerrado e a Floresta Estacional e de ampla ocorrência nos estados de TO, MT, GO, MG, SP e MS.

A faveira é uma árvore de porte médio a alto que, além de sombra, produz casca e frutos com propriedades medicinais, fornecendo também madeira muito dura usada na construção civil (BARROS, 1982; DIAS, 1993; JUNQUEIRA et al., 2000; LORENZI, 1992; VALE et al., 2010) e que é considerada pelos proprietários como uma reserva econômica para o futuro.

A manutenção ou plantio de árvores nativas em pastagens pode resultar em benefícios para os diferentes componentes do agroecossistema, como é o caso do microclima, plantas forrageiras e animais. Os atributos físicos e químicos dos solos, muitas vezes degradados, bem como a atividade biológica também são beneficiados visto que ocorrerá um aporte maior de matéria orgânica e ciclagem de nutrientes (MENDONÇA et al., 2001). Além de garantir condições ambientais mais propícias às suas pastagens e criações, o pecuarista pode obter também vantagens econômicas pelo suprimento de madeira disponível para uso próprio ou comércio, sem abandonar a sua vocação para a pecuária.

Dentro da gama de pesquisas existentes sobre os sistemas que apresentam como componente arbóreo o $P$. emarginatus, são raros os trabalhos referentes aos aspectos dendrométricos. Não se conhece para essa espécie, dentro desses SSP intuitivos, as relações hipsométricas e volumétricas, bem como as interações entre as copas e os diâmetros das árvores.

Cerne, Lavras, v. 19, n. 1, p. 141-149, jan./mar. 2013
Essas relações permitem prever o espaço exigido pelas árvores para o seu desenvolvimento, fazer inferências sobre a estabilidade, vitalidade e produtividade de cada indivíduo, avaliar as relações de concorrência e auxiliar na elaboração de modelos de crescimento florestal (DURLO et al., 2004).

Nesse contexto, objetivou-se, neste estudo, avaliar por meio de regressão as relações alométricas possíveis entre diâmetro a 1,3 m de altura, altura de copa, altura de fuste, altura total, diâmetro e volume cilíndrico de copa, em um SSP P. emarginatus x Brachiaria decumbens $\mathrm{x}$ bovinos de corte.

\section{MATERIAL E MÉTODOS}

A coleta de dados foi realizada durante o mês de setembro de 2008, na Fazenda Gogó da Ema (21 ${ }^{\circ} 07^{\prime} 32.85^{\prime \prime}$ S e 55 47' 45.53" W, $276 \mathrm{~m}$ de altitude), distante $4 \mathrm{~km}$ da cidade de Nioaque - MS, em uma área de pastagem de 17 ha. O clima é classificado como tropical (Aw), com estação seca de inverno em transição com temperado úmido (Cfa) com verão quente, segundo classificação climática de Köppen. A precipitação anual varia de 1500 a 1750 mm, o excedente hídrico anual é de 800 a $1200 \mathrm{~mm}$ (durante 5 a 6 meses), deficiência hídrica de 350 a $500 \mathrm{~mm}$ durante 4 meses (INSTITUTO BRASILEIRO DE GEOGRAFIA E ESTATÍSTICA - IBGE, 2000) e temperatura média anual de 23 a $26^{\circ} \mathrm{C}$. A área está sobre um Latossolo Vermelho Distrófico, cuja composição granulométrica na camada de 0 a $20 \mathrm{~cm}$, determinada segundo Claessen (1997), foi $98 \mathrm{~g} \mathrm{~kg}^{-1}$ de argila, $13 \mathrm{~g} \mathrm{~kg}^{-1}$ de silte e $889 \mathrm{~g} \mathrm{~kg}^{-1}$ de areia.

A área, originalmente sob vegetação de Cerrado em transição com Floresta Estacional Semidecidual é atualmente ocupada com pastagem de B. decumbens, destinada à pecuária de corte, sombreada por espécies arbóreas, predominantemente $P$. emarginatus (sucupirabranca). Segundo relatos do proprietário, a área do sistema silvipastoril nunca recebeu adição de corretivos ou fertilizantes (Figura 1).

Para as análises, foram coletados dados de altura total (At), altura do fuste (Af), circunferência à altura do peito (CAP) e diâmetro de copa (Dc), de uma amostra composta por 91 indivíduos adultos, de variadas dimensões, aleatoriamente dispersos na área em estudo. Para compor a amostra, foi feita a seleção das árvores partindo-se de um indivíduo escolhido ao acaso, seguindo para a vizinha mais próxima e assim sucessivamente, independente da CAP observada, até que se atingisse a divisa da área. 
A

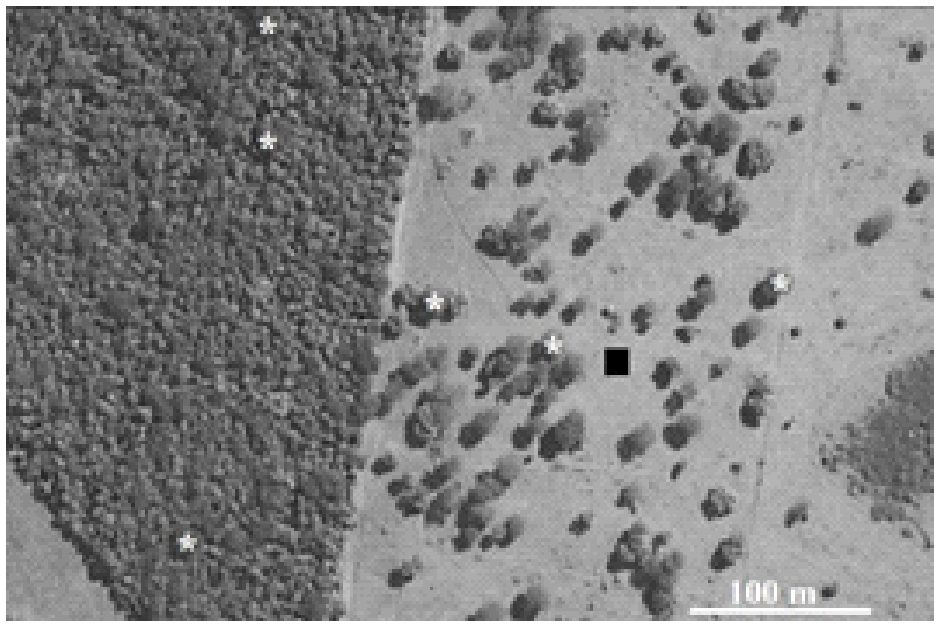

B

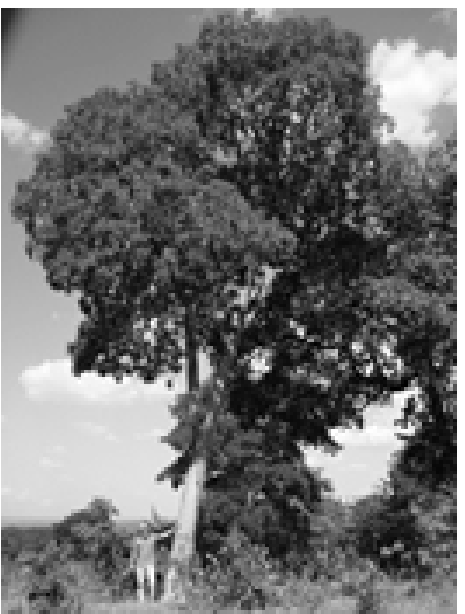

Figura 1 - A - Imagem do satélite GeoEye-1 (02/04/2007) - esquerda: vegetação típica original; direita: parte do SSP analisado; - ponto das coordenadas $21^{\circ} 07^{\prime} 32.85^{\prime \prime} \mathrm{S}$ e $55^{\circ} 47^{\prime} 45.53^{\prime \prime} \mathrm{W}$; * - alguns exemplares de P. emarginatus. Fonte: Google Earth (2010). B - Fotografia de um exemplar de P. emarginatus representativo da espécie em área de Sistema Silvipastoril, Fazenda Gogó da Ema, Município de Nioaque - MS. Fonte: Pezzoni (2010).

Figure 1 - A - Image of satellite GeoEye.1 (02/04/2007) - left: original typical vegetation; right: part of SPS; - point of coordenates $21^{\circ} 07^{\prime} 32.85^{\prime \prime} S$ and 55 47' 45.53" W; * - some specimens of P. emarginatus. (GOOGLE EARTH, 2010). B Specimem of P. emarginatus representative of specie in SPS, Gogó da Ema Farm, municipal district of Nioaque - MS. (PEZZONI, 2010).

At e Af foram obtidas por trigonometria a partir de ângulos verticais e distâncias horizontais tomados a partir de um clinômetro, deduzindo-se, em seguida, a altura da copa (Ac) pela subtração das variáveis At e Af. Aárea de copa foi calculada tomando-se como base o diâmetro médio das copas dos indivíduos, sendo este o resultado da média entre duas medidas perpendiculares da projeção das copas sobre o solo. O volume cilíndrico de copa (Vc) foi determinado peloproduto entre a área da base da copa e a Ac. A CAP, medida com auxílio de fita métrica, foi, posteriormente, transformada em diâmetro a 1,3 m de altura (DAP).

Os dados obtidos foram submetidos a análises de regressões simples ou múltiplas, utilizando-se modelos lineares, linearizados ou não lineares, conforme Caldeira et al. (2003), Couto e Bastos (1987), Curtis (1967) e Soares et al. (2004) (Tabelas 1 e 2).

Testes de aderência de Kolmogorov-Smirnov (KS) ( $p \geq 0,05)$, Lilliefors ( $p \geq 0,05)$ e teste $W$ de ShapiroWilk (SW) para verificação da normalidade das variáveis dependentes foram aplicados a $\mathrm{P} \leq 0,01$, considerandose que a variável segue a distribuição normal quando a distribuição dos dados foi não significativa em pelo menos dois deles.

A escolha das variáveis que deveriam ser utilizadas como regressoras foi feita com base no coeficiente de correlação ( $\mathrm{r} \geq 0,50)$, o que limitou as análises a: At x DAP $(\mathrm{r}=0,79 * *)$, At x Ac $\left(\mathrm{r}=0,88^{* *}\right), \operatorname{Ac} \mathrm{x}$ $\operatorname{DAP}(\mathrm{r}=0,72 * *), \operatorname{Vc} x \operatorname{DAP}(\mathrm{r}=0,72 * *), \operatorname{Vc} x \operatorname{At}(\mathrm{r}=0,68 * *)$, $\operatorname{Vc} x \operatorname{Ac}(\mathrm{r}=0,68), \operatorname{Dc} \times \operatorname{DAP}(\mathrm{r}=0,74 * *)$.

Para a seleção dos modelos foram considerados como critérios a significância dos coeficientes de regressão pelo teste t a 5\%, o coeficiente de determinação ajustado $\left(\mathrm{R}^{2}\right)$, o erro padrão residual (EPR) e a distribuição gráfica dos resíduos em percentagem. Foi feita uma triagem inicial entre os modelos identificados na literatura e aqueles com $\mathrm{R}^{2}<0,5$ foram desconsiderados nas análises. O EPR foi recalculado sempre que os ajustes contemplaram modelos linearizados, cujas variáveis dependentes tenham sido transformadas: EPR= $\sqrt{\sum_{\mathrm{n}=1}^{\mathrm{n}}\left(\mathrm{v}_{\mathrm{obs}}-\mathrm{v}_{\mathrm{est}}\right)^{2} /[\mathrm{n}-(\mathrm{p}-1)]}$, em que $\mathrm{v}_{\text {obs }}$ e $\mathrm{v}_{\text {est }}$ são os valores observados e estimados, $n=n^{\circ}$ de dados e $p=n^{\circ}$ de coeficientes de cada modelo. 
Tabela 1 - Modelos testados tendo como variáveis dependentes a altura total e de copa, o volume cilíndrico de copa e o diâmetro de copa, em função de uma variável independente para amostras de $P$. emarginatus em um sistema silvipastoril.

Table 1 - Tested models using as dependent variables the total height and crown height, the cylindrical volume of crown and the crown diameter, in function of one independent variable for samples of P. emarginatus in a silvopastoral system.

\begin{tabular}{|c|c|}
\hline Altura total (y) $f$ DAP (x) & Altura de copa $(\mathrm{y}) f$ DAP $(\mathrm{x})$ \\
\hline (1) $y=a+b \cdot x+c \cdot x^{2}$ (parabólico) & (9) $y=a+b \cdot x$ \\
\hline (2) $\operatorname{Ln}(y)=a+b \cdot \operatorname{Ln}(x)$ (Stoffels, potência) & $(10) \mathrm{y}=\mathrm{a} \cdot(\mathrm{x} / 1+\mathrm{x})^{\mathrm{b}}$ \\
\hline (3) $\operatorname{Ln}(y)=a+b \cdot x^{-1}$ (Curtis, exponencial) & Altura de copa (y) $f$ Altura total (x) \\
\hline (4) $y=a+b \cdot x$ (reta) & (11) $y=a+b \cdot x$ \\
\hline (5) $\mathrm{y}=\mathrm{a}+\mathrm{b} \cdot\left(1 / \mathrm{x}^{2}\right)$ (hiperbólico) & Volume cilíndrico de copa (y) $f$ DAP (x) \\
\hline (6) $y=1 /\left[a+b \cdot(1 / x)+c \cdot(1 / x)^{2}\right]($ Prodan $)$ & (12) $y=a+b \cdot x$ \\
\hline (7) $y=1 /[a+b .(1 / x)]^{3}$ (Petterson) & Diâmetro de copa $f$ DAP (x) \\
\hline (8) $y=(x / a+b \cdot x)^{2}$ & (13) $y=a+b \cdot x$ \\
\hline
\end{tabular}

Tabela 2 - Modelos testados tendo como variáveis dependentes o volume cilíndrico de copa em função de duas variáveis independentes para amostras de P. emarginatus em sistema silvipastoril.

Table 2 - Tested models using as dependent variables the cylindrical volume of crown, the cylindrical volume of crown in function of two independent variable for samples of P. emarginatus in a silvopastoral system.

\begin{tabular}{|c|c|c|}
\hline \multirow[b]{2}{*}{ Modelos } & \multicolumn{2}{|c|}{ Ordem dos modelos no texto para: } \\
\hline & $\begin{array}{c}\text { Volume de copa }(\mathrm{y}) f\left[\mathrm{DAP}\left(\mathrm{x}_{1}\right) ;\right. \\
\text { Altura total }\left(\mathrm{x}_{2}\right)\end{array}$ & $\begin{array}{c}\text { Volume de copa }(\mathrm{y}) f\left[\mathrm{DAP}\left(\mathrm{x}_{1}\right)\right. \\
\left.\text { Altura do fuste }\left(\mathrm{x}_{2}\right)\right]\end{array}$ \\
\hline $\mathrm{y}=\mathrm{a}+\mathrm{b} \cdot\left(\mathrm{x}_{1}^{2} \cdot \mathrm{x}_{2}\right),($ Spurr $)$ & $(1)$ & (7) \\
\hline $\operatorname{Ln}(y)=a+b \cdot\left(x_{1}^{2} \cdot x_{2}\right)$, Spurr & (2) & $(8)$ \\
\hline $\operatorname{Ln}(y)=a+b \cdot \operatorname{Ln}\left(x_{1}^{2} \cdot x_{2}\right),($ Spurr $)$ & (3) & (9) \\
\hline $\begin{aligned} \operatorname{Ln}(y)= & a+b \cdot \operatorname{Ln}\left(x_{1}\right)+c \cdot \operatorname{Ln}\left(x_{2}\right) \\
& (\text { Schumacher-Hall })\end{aligned}$ & (4) & $(10)$ \\
\hline $\mathrm{y}=\mathrm{a} \cdot\left(\mathrm{x}_{1}^{\mathrm{b}}\right) \cdot \mathrm{x}_{2}^{\mathrm{c}}$ & (5) & $(11)$ \\
\hline $\mathrm{y}=\mathrm{a} \cdot\left(\mathrm{x}_{1}^{2} \cdot \mathrm{x}_{2}\right)^{\mathrm{b}}$ & (6) & (12) \\
\hline
\end{tabular}

\section{RESULTADOS E DISCUSSÃO}

O primeiro procedimento de análise de dados foi a verificação da normalidade das distribuições dos dados referentes às variáveis dependentes. Segundo os testes aplicados, todas elas apresentam distribuição normal, o que evitou a aplicação de transformações e garantiu que a distribuição normal padrão (z) de cada uma apresente $\mu=0$ e $\sigma=1$ (Tabela 3).

A partir da constatação da normalidade das distribuições das variáveis dependentes, foram calculadas a amplitude e variação dos parâmetros com base na amostra de 91 indivíduos (Tabela 4). Constatou-se que há uma grande faixa de variação dos dados, especialmente no que se refere ao DAP $(29,9$ a 76,4 cm) e à At (12,2 a 29,4 m), o que contribuiu para a aplicabilidade das equações ajustadas e para a precisão das médias estimadas.

Procedeu-se, então, ao ajuste das equações, dividindose os modelos em dois grupos: aqueles que contemplam apenas uma variável regressora e os que operam com duas.

\subsection{Ajustes para uma única variável regressora}

No contexto dos ajustes para uma única variável regressora (DAPe At, Tabela 1), foram estimados os coeficientes dos diferentes modelos testados (Figura 2).

\section{Cerne, Lavras, v. 19, n. 1, p. 141-149, jan./mar. 2013}


Tabela 3 - Teste de aderência para normalidade de Kolmogorov-Smirnov (K-S) ( $p \geq 0,05)$, Lilliefors ( $p \geq 0,05)$ e teste W de ShapiroWilk (SW) para as variáveis dependentes de amostra de P. emarginatus em sistema silvipastoril.

Table 3 - Normality tests of Kolmogorov-Smirnov $(K-S)(p \geq 0,05)$, Lilliefors $(p \geq 0,05)$ and W test of Shapiro-Wilk $(S W)$ for dependents variables of sample of $P$. emarginatus in a silvopastoral system.

\begin{tabular}{|c|c|c|c|c|c|c|}
\hline Variável* & K-S (D) & $\mathrm{K}-\mathrm{S} \mathrm{p}(\mathrm{D})$ & Lilliefors & S-W (W) & $\mathrm{S}-\mathrm{W} \mathrm{p}(\mathrm{W})$ & Distribuição \\
\hline $\mathrm{DAP}(\mathrm{cm})$ & 0,08816 & $>0,20$ & $<0,10$ & 0,97849 & 0,13689 & Normal \\
\hline $\operatorname{Af}(m)$ & 0,08315 & $>0,20$ & $>0,15$ & 0,96725 & 0,02157 & Normal \\
\hline At (m) & 0,05639 & $>0,20$ & $>0,20$ & 0,98957 & 0,69252 & Normal \\
\hline Ac (m) & 0,05959 & $>0,20$ & $>0,20$ & 0,96998 & 0,03362 & Normal \\
\hline Dc (m) & 0,05989 & $>0,20$ & $>0,20$ & 0,98298 & 0,28190 & Normal \\
\hline $\mathrm{Vc}\left(\mathrm{m}^{3}\right)$ & 0,09511 & $>0,20$ & $<0,05$ & 0,94617 & 0,00091 & Normal \\
\hline
\end{tabular}

* DAP - diâmetro a 1,3 m de altura, Af - altura do fuste, At - altura total, Ac - altura de copa, Dc - diâmetro de copa, Vc - volume cilíndrico de copa.

Tabela 4 - Amplitude e variação dos parâmetros da amostra de 91 indivíduos de P. emarginatus em sistema silvipastoril.

Table 4-Amplitude and variation of the parameters in a sample of 91 specimens of P. emarginatus in a silvopastoral system.

\begin{tabular}{lcccccccc}
\hline Variável* & Média & $\begin{array}{c}\text { IC** } \\
(-95 \%)\end{array}$ & $\begin{array}{c}\text { IC } \\
(95 \%)\end{array}$ & Valor mínimo & $\begin{array}{c}\text { Valor } \\
\text { máximo }\end{array}$ & $\begin{array}{c}\text { Desvio } \\
\text { padrão }\end{array}$ & $\begin{array}{c}\text { CV*** } \\
(\%)\end{array}$ & $\begin{array}{c}\text { Erro } \\
\text { padrão }\end{array}$ \\
\hline DAP $(\mathrm{cm})$ & 50,2 & 48,0 & 52,4 & 29,9 & 76,4 & 10,65 & 21,2 & 1,12 \\
Af $(\mathrm{m})$ & 6,3 & 5,9 & 6,7 & 3,3 & 10,2 & 1,79 & 28,5 & 0,19 \\
At (m) & 20,5 & 19,8 & 21,2 & 12,2 & 29,4 & 3,49 & 17,0 & 0,37 \\
Ac (m) & 14,2 & 13,5 & 14,9 & 7,6 & 24,5 & 3,50 & 24,7 & 0,37 \\
Dc (m) & 13,2 & 12,7 & 13,8 & 8,35 & 20,55 & 2,60 & 19,7 & 0,27 \\
Vc $\left(\mathrm{m}^{3}\right)$ & 142,4 & 130,6 & 154,1 & 54,76 & 331,68 & 56,55 & 39,7 & 5,93 \\
\hline
\end{tabular}

* Definidos anteriormente, ** IC - intervalo de confiança, *** CV - coeficiente de variação.

Alguns dos modelos que tinham At como variável dependente foram excluídos das discussões, por apresentarem algum tipo de viés, como foram os casos dos modelos 3, 7 e 8 . O modelo 8 foi ajustado com sucesso por Caldeira et al. (2003) em araucária, tendo sido selecionado para uso no oeste do Paraná. No entanto, quando aplicado sobre os dados aqui utilizados, a equação gerou superestimativas para os valores menores e subestimativas nos maiores.

Os modelos 1,5 e 6 apresentaram $\mathrm{R}^{2}$ abaixo de 0,5 ou os coeficientes não significativos pelo teste $\mathrm{t}(\mathrm{P} \leq 0,05)$, demonstrando que as equações ajustadas a partir deles são inapropriadas para a estimativa das alturas totais de $P$. emarginatus nos SSP analisados.

Os modelos 2 e 4, com todos os coeficientes significativos, não apresentaram tendenciosidades nas estimativas de At para qualquer dimensão de DAP, segundo a distribuição gráfica dos resíduos. Os valores de EPR variaram de 1,8 a 2,1\% e os de $\mathrm{R}^{2}$ de 0,62 a 0,63 , respectivamente. Assim, por esses critérios, as duas equações ajustadas apresentaram níveis de precisão semelhantes.

$\mathrm{O}$ modelo 2, uma potência em sua forma original $\left[y=\beta_{0}^{\prime} \cdot x^{\beta_{1}}\right]$, confere apelo biológico, sendo de uso generalizado nas ciências florestais. Nas relações entre diâmetro e altura, expressa o menor diâmetro necessário para sustentar uma árvore sem ruptura em função de uma potência da altura, segundo O'Brien et al. (1995). Por essa constatação, conclui-se que a equação 2 é a mais adequada para pequenas extrapolações, já que, embora tenha apresentado $\mathrm{R}^{2}$ semelhante ao modelo 4, destaca-se com o menor EPR.

Estudo de Macera e Andrade Filho (2010), que analisaram quatro das equações mais utilizadas em hipsometria, apontou que o modelo 2 (Stoffels) foi o que melhor se ajustou aos dados, com a suposição de que a variável resposta tenha distribuição normal. Conforme se observa na Tabela 3, a variável dependente At usada apresenta esta condição.

Cerne, Lavras, v. 19, n. 1, p. 141-149, jan./mar. 2013 


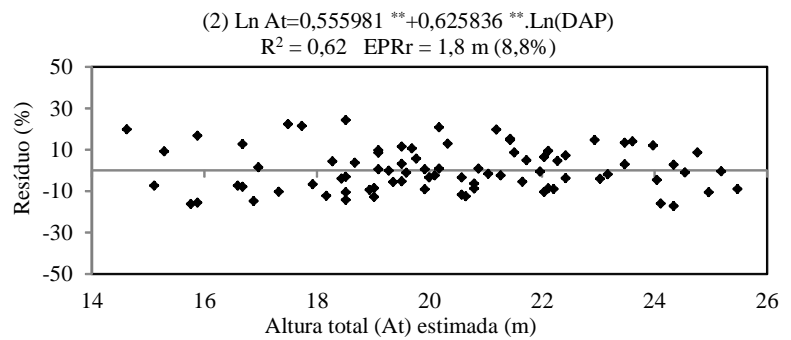

(4) $\mathrm{At}=7,475265^{* *}+0,253967^{* *}$.DAP

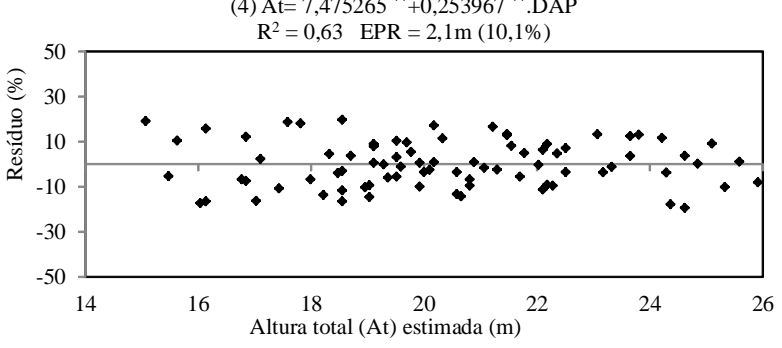

(11) $\mathrm{Ac}=-3,13311^{* *}+0,85156^{* *}$. At $\mathrm{R}^{2}=0,80 \quad \mathrm{EPR}=1,6 \mathrm{~m}(10,7 \%)$
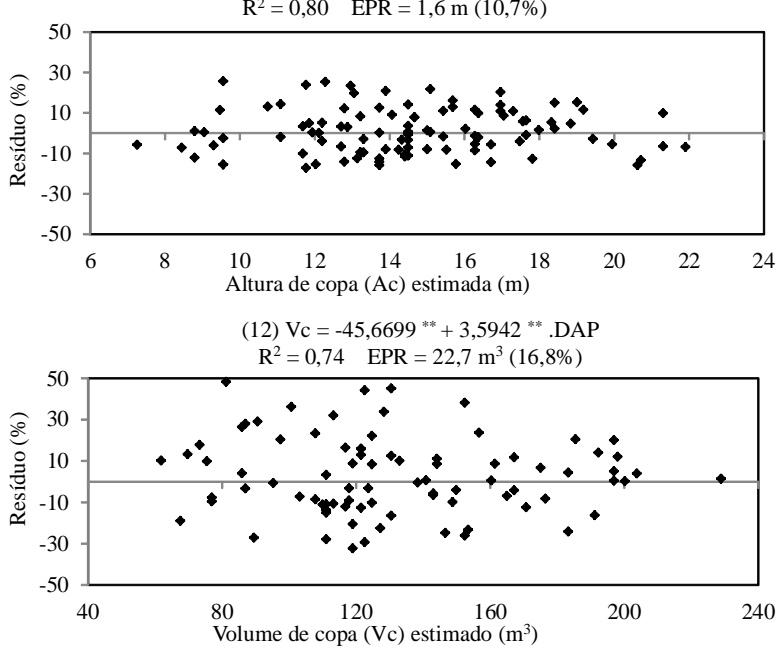

(13) $\mathrm{Dc}=2,671313^{* *}+0,209866^{* *}$.DAP

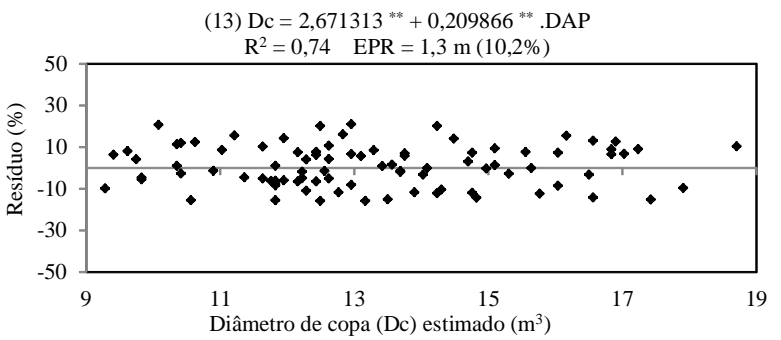

Figura 2 - Equações ajustadas, estatísticas de precisão e gráficos de resíduos da amostra de $P$. emarginatus em sistema silvopastoril, para uma variável independente.

Figure 2-Adjusted equations, statistics of precision and graphs of residues of $P$. emarginatus sample in a silvopastoral system, for one independent variable.

Cerne, Lavras, v. 19, n. 1, p. 141-149, jan./mar. 2013
Os modelos que estimam Ac (modelos 9 e 10) em função do DAP tiveram $\mathrm{R}^{2}<0,5 \mathrm{e}$, por isso, foram excluídos das análises. O modelo 11 (Figura 2) apresentou melhores medidas de precisão da regressão. Os baixos valores de $\mathrm{R}^{2}$ encontrados para os modelos 9 e 10, provavelmente estão relacionados à dispersão dos dados e com o menor número de pontos de Ac nas extremidades.

Essa foi uma situação difícil de ser resolvida durante a coleta de dados, já que as árvores presentes no sistema existem no local desde que a área foi convertida de vegetação natural para SSP. Não houve, portanto, regeneração natural e foram mantidos apenas indivíduos adultos, evitando-se, na visão dos produtores, superpopulação arbórea que poderia prejudicar a produtividade das pastagens.

Esse critério é comum na região onde, na execução da retirada da vegetação nativa para formar pastagens, mantêm-se apenas indivíduos adultos. Isso prejudicou, certamente, a precisão das estimativas para os menores valores que, todavia, na prática não existem.

Os ajustes de equações para estimar Ac $f$ At, e Vc $f$ DAP e Dc $f$ DAP limitaram-se ao modelo da linha reta, tendo em vista que a dispersão dos dados demonstrou claramente essa tendência. As equações ajustadas apresentaram significância para os coeficientes estimados, $\mathrm{R}^{2} \geq 0,74$, além de não demonstrarem tendenciosidade por meio dos gráficos de resíduos (Figura 2).

De todos os ajustes feitos em função de uma única variável independente, Ac $f$ At apresentou os melhores resultados, com $\mathrm{R}^{2}=0,80$ e $\mathrm{EPR}=10,7 \%$.

\subsection{Ajustes para duas variáveis regressoras}

Nesta seção o objetivo foi o ajuste de equações para estimar o Vc $f$ (DAP;At) e Vc $f$ (DAP;Af) (Figura 3), utilizando-se os modelos da Tabela 2.

Durante os ajustes, foram descartados os modelos que apresentaram coeficientes não significativos ou $\mathrm{R}^{2} \leq 0,5$, como foi o caso do modelo 5 para Vc $f$ (DAP;At) e de todos os modelos ajustados para Vc $f$ (DAP;Af). As imprecisões dos modelos testados para Vc $f$ (DAP;Af) motivaram a exclusão dessa relação para estimar o volume de copa para $P$. emarginatus, nas condições do SSP aplicado no MS, dentro da amplitude de dados coletados.

Restaram, portanto, os modelos 1, 2, 3, 4 e 6 da relação Vc $f$ (DAP;At), apresentados na Figura 3. Embora sejam modelos tradicionalmente utilizados para a estimativa de volume de fuste, aqui foram aplicados para estimar o 

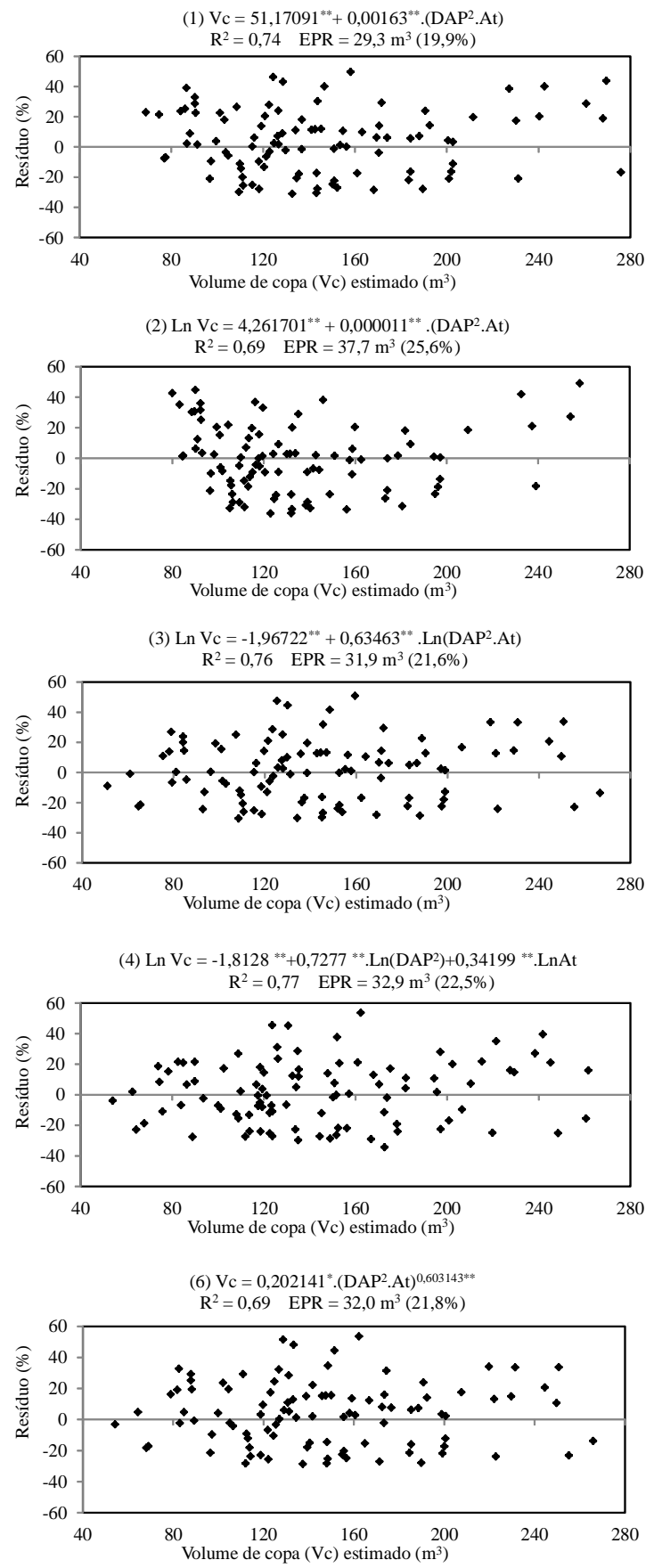

Figura 3 - Equações ajustadas, estatísticas de precisão e gráficos de resíduos da amostra de $P$. emarginatus em sistema silvipastoril, para duas variáveis independentes.

Figure 3 -Adjusted equations, statistics of precision and graphs of residues of $P$. emarginatus sample in a silvopastoral system, for two independent variables. volume cilíndrico de copa. Não apresentam tendenciosidade e resultaram em coeficientes de determinação razoáveis $(0,69$ a 0,77$)$, já que a sua variação dentro da amostra foi a maior obtida (Tabela 4). Esses modelos também foram testados por Machado et al. (2008) em bracatinga (Mimosa scabrella Bentham) para estimar o volume do fuste, onde obtiveram os maiores $\mathrm{R}^{2}$ e os menores EPR. O modelo 4 foi testado em árvores de candeia (Eremanthus erythropappus (DC.) MacLeish) tendo sido o de melhor ajuste dentre os modelos avaliados para estimar o volume do fuste (SCOLFORO et al., 2004). Os modelos 1, 3 e 4 , em ordem de prioridade, destacaram-se como os melhores modelos para estimar o Vc $f$ (DAP;At).

É possível que melhores estimativas e precisão possam ser obtidas se forem avaliados os volumes reais das copas. Apesar das dificuldades de realizar essa tarefa, é possível trabalhar a modelagem de Vc a partir de fotografias, conforme propõem Durlo et al. (2004), ou com estimativas obtidas a partir dos ângulos e raios formados pela projeção da copa da árvore no solo (SCOLFORO; THIERSCH, 2004).

\section{CONCLUSÕES}

O modelo de Stoffels foi o mais adequado para estimar a altura total em função do diâmetro a 1,3 m de altura.

A altura de copa em função do diâmetro a 1,3 m de altura demonstrou ser uma relação inapropriada para o sistema de produção avaliado.

Para as estimativas de altura de copa em função da altura total, volume cilíndrico de copa em função do diâmetro a 1,3 m de altura e diâmetro de copa em função do diâmetro a 1,3 m de altura, o modelo da reta resultou nos melhores níveis de precisão.

O volume cilíndrico de copa em função do diâmetro a 1,3 m de altura e da altura total foi estimado com bons níveis de precisão, a partir do modelo de Spurr [Vc = 51,17091+0,00163.(DAP ${ }^{2}$.At) $]$.

Os resultados obtidos facilitam o planejamento de novos estudos referentes à influência das árvores de sucupira sobre as propriedades do solo e qualidade da forragem, considerando, particularmente, que os melhores modelos de regressão foram aqueles relacionados às variáveis altura e copa, as quais influenciam diretamente no sombreamento e produção de serapilheira, permitindo ainda simulações relacionadas à densidade ideal de árvores por unidade de área.

Cerne, Lavras, v. 19, n. 1, p. 141-149, jan./mar. 2013 


\section{AGRADECIMENTOS}

Os autores agradecem aos proprietários da Fazenda Gogó da Ema (Nioaque - MS), na pessoa de Álvaro Eugênio Dalamarta Domingues, pela cessão da área para pesquisa e a estadia na sede durante a coleta de dados.

\section{REFERÊNCIAS}

BARROS, M. A. G. Flora medicinal do Distrito Federal. Revista Brasil Florestal, Rio de Janeiro, v. 12, p. 35-45, 1982.

CALDEIRA, M. V. W.; SCHUMACHER, M. V.; SCHEEREN, L. W.; WATZLAWICK, L. F. Relação hipsométrica para Araucaria angustifolia (Bert.) O. Ktze na região oeste do estado do Paraná. Revista Acadêmica: Ciências Agrárias e Ambientais, Curitiba, v. 1, n. 2, p. 7988, 2003.

CLAESSEN, M. E. C. Manual de métodos de análise de solo. 2. ed. Rio de Janeiro: EMBRAPA Solos, 1997.

(Documentos, 1).

COUTO, H. T. Z.; BASTOS, N. L. M. Modelos de equações de volume e relações hipsométricas para plantaçõoes de Eucalyptus no Estado de São Paulo. IPEF, Piracicaba, v. 37, p. 33-44, 1987.

CURTIS, R. O. Height-diameter and height-diameter-age equations for second-growth Douglas-fir. Forest Science, Bethesda, v. 13, n. 4, p. 365-375,1967.

DANIEL, O.; COUTO, L.; VITORINO, A. C. T. Sistemas agroflorestais como alternativas sustentáveis à recuperação de pastagens degradadas. In: SIMPÓSIO SUSTENTABILIDADE DA PECUÁRIA DE LEITE NO BRASIL, 1., 1999, Goiânia. Anais... Juiz de Fora: EMBRAPA Gado de Leite, 1999. p. 151-170.

DIAS, F. L. Estudo da genotoxicidade in vivo e in vitro dos cercaricidas naturais óleo de sucupira e cremantina em células de mamíferos. 1993. 105 f. Tese (Doutorado em Medicina) - Faculdade de Medicina de Ribeirão Preto, Ribeirão Preto, 1993.

DURLO, M. A.; SUTILI, F. J.; DENARDI, L. Modelagem da copa de Cedrela fissilis Vellozo. Ciência Florestal, Santa Maria, v. 14, n. 2, p. 79-89, mar./abr. 2004.
GOOGLE EARTH. Imagem de satélite: coordenadas $21^{\circ} 07^{\prime}$ 32.85" S e 5547'45.53"W. Disponível em: <http:// www.google.com/earth/index.html>. Acesso em: 10 out. 2010.

INSTITUTO BRASILEIRO DE GEOGRAFIA E ESTATÍSTICA. Banco de dados agregados censo 2000. Disponível em: <http://www.sidra.ibge.gov.br/bda/extveg/ default.asp>. Acesso em: 15 ago. 2009.

JUNQUEIRA, N. T. V.; NASCIMENTO, A. C.; PINTO, A. C. Q.; RAMOS, U. H. V.; PIO, R.; RANGEL, L. E. P.; SILVA, J. A.; FIALHO, J. F. Efeito do extrato dos frutos de sucupirabranca (Pterodon pubescens Benth.) e de outros produtos naturais no controle de doenças de manga na pós-colheita. In: CONGRESSO BRASILEIRO DE DEFENSIVOS AGRÍCOLAS NATURAIS, 1., 2000, Fortaleza. Anais... Fortaleza: UFC, 2000. p. 36.

LORENZI, H. Árvores brasileiras: manual de identificação e cultivo de plantas arbóreas nativas do Brasil. Nova Odessa: Plantarum, 1992. 352 p.

MACERA, M. A. C.; ANDRADE FILHO, M. G. Ajuste de relações hipsométricas com heterocedasticidade usando GAMLSS. In: SIMPÓSIO NACIONAL DE PROBABILIDADE E ESTATÍSTICA, 19., 2010, São Pedro. Resumos... São Pedro: USP, 2010. p. 1-6.

MACHADO, S. A.; FIGURA, M. A.; SILVA, L. C. R.; TÉO, S. J.; STOLLE, L.; URBANO, E. Modelagem volumétrica para bracatinga (Mimosa scabrella) em povoamentos da Região Metropolitana de Curitiba. Pesquisa Florestal Brasileira, Colomgo, n. 56, p. 17-29, 2008.

MENDONÇA, E. S.; LEITE, L. F. C.; FERREIRA NETO, P. S. Cultivo de café em sistema agroflorestal: uma opção para recuperação de solos degradados. Revista Árvore, Viçosa, v. 25, n. 3, p. 375-383, maio/jun. 2001.

MITTERMEIER, R. A.; GIL, P. R.; HOFFMAN, M.; PILGRIM, J.; BROOKS, T.; MITTERMEIER, C. G.; LAMOREUX, J.; FONSECA, G. A. B. Hotspots revisited: earth's biologically richest and most endangered terrestrial ecoregions. Chicago: Chicago Press, 2005. 392 p.

O'BRIEN, S. T.; HUBBELL, S. P.; SPIRO, P.; CONDIT, R.; FOSTER, R. B. Diameter, height, crown, and age relationships in eight neotropical tree species. Ecology, Durham, v. 76, n. 6, p. 1926-1939, 1995. 
PEZZONI, T. Influência de Pterodon emarginatus Vogel sobre atributos físicos e químicos do solo e valor nutritivo de Brachiaria decumbens Stapf em sistema silvipastoril. 2010. 43 f. Dissertação (Mestrado em Produção Vegetal) - Universidade Federal da Grande Dourados, Dourados, 2010.

SCOLFORO, J. R. S.; PÉREZ, J. F.; MELLO, J. M.; OLIVEIRA, A.D.; CAMOLESI, J. F.; BORGES, L. F. R.; ACERBI JÚNIOR, F. W. Estimativas de volume, peso seco, peso de óleo e quantidade de moirões para a candeia (Eremanthus erythropappus (DC.) MacLeish). Cerne, Lavras, v. 10, n. 1, p. 87-102, 2004.
SCOLFORO, J. R. S.; THIERSCH, C. R. Biometria florestal: medição, volumetria e gravimetria. Lavras: UFLA/ FAEPE, 2004.

SOARES, T. S.; SCOLFORO, J. R. S.; FERREIRA, S. O.; MELLO, J. M. D. Uso de diferentes alternativas para viabilizar a relação hipsométrica no povoamento forestal. Revista Árvore, Viçosa, v. 28, n. 6, p. 845-854, nov./dez. 2004.

VALE, A. T.; DIAS, I. S.; SANTANA, M. A. E. Relações entre propriedades químicas, físicas e energéticas da madeira de cinco espécies de cerrado. Ciência Florestal, Santa Maria, v. 20, n. 1, p. 137-146, jan. 2010. 
